\title{
Weak Disorder Expansions for the Anderson Model on a One Dimensional Strip at the Center of the Band
}

\section{Athanasios Speis}

Department of Mathematics, University of Michigan, Ann Arbor, MI 48109, USA

Received March 13, 1991; in revised form September 18, 1991

Abstract. We study the asymptotic behavior of the averaged diagonal matrix elements of the Greens kernel for the Anderson Model on a one-dimensional strip and for a set of special energies close to the center of the band.

\section{Introduction}

Let $\ell$ be a positive integer and let $\mathscr{D}_{\ell}$ be the one dimensional lattice strip of with $\ell$, i.e., $\mathscr{Z}_{\ell}=\mathbb{Z} \times\{1, \ldots, \ell\}$, where $\mathbb{Z}$ is the set of all integers.

The Anderson model [1] on $\mathscr{D}_{\ell}$ is given by the random Hamiltonian $H_{\lambda}=-\frac{1}{2} \Delta+\lambda V$ on $\ell^{2}\left(\mathscr{D}_{\ell}\right)$, where

with

$$
(\Delta u)(x) \sum_{y \in \mathscr{Q} \ell}(\Delta)_{x, y} u(y)
$$

$$
(\Delta)_{x, y}= \begin{cases}1 & \text { if } x-y \in\{(0,1),(-1,0),(1,0),(0,-1)\} \\ 0 & \text { otherwise }\end{cases}
$$

and

$$
(V u)(x)=V(x) u(x),
$$

where $\{V(x)\}_{x \in \mathscr{D} \ell}$ are i.i.d. real random variables with common distribution $\mu$ whose characteristic function will be denoted by $h$ and $\lambda$ is a real number.

Let $m$ be a positive integer, $A^{m}$ be the discrete rectangle $[-m, m] \times\{1,2, \ldots, \ell\}$ and $H_{m, \lambda}$ denote the $H_{\lambda}$ restricted to $\ell^{2}\left(\Lambda_{\ell}\right)$ with boundary conditions $u(x)=0$ for all $x \notin \Lambda^{m}$. Let $x, y \in \mathscr{D}_{\ell}, \eta>0$ and let $\delta_{x}, \delta_{y} \in \ell^{2}\left(\mathscr{D}_{\ell}\right)$ be the delta functions at the points $x$ and $y$ respectively. We shall use the notations

$$
\begin{gathered}
G_{m}^{\lambda}(x, y, E+i \eta)=\left\langle\delta_{x}\left|\frac{1}{H_{m, \lambda}-E-i \eta}\right| \delta_{y}\right\rangle, \\
J_{\lambda}^{j}(E+i \eta)=\lim _{m \rightarrow+\infty} \mathbb{E}\left\{G_{m}^{\lambda}((0, j),(0, j), E+i \eta)\right\},
\end{gathered}
$$

where $j=1, \ldots, \ell$ and $E\{\cdot\}$ denotes the expectation with respect to the disorder. 
It is a consequence of the "de la Vallée Poussin" theorem [2] that the $\lim _{\eta \downarrow 0} J_{\lambda}^{j}(E+i \eta)$ exists for almost all $E \in \mathbb{R}$ and all $\lambda \neq 0$. Under some mild regularity conditions for $h$ Klein, Lacroix and Speis [3] have shown that the limit above actually exists for all $E \in \mathbb{R}$ and it is a smooth function of $E$ for all $\lambda \neq 0$.

The boundary value of $J_{\lambda}^{j}(E+i \eta)$ described above contains a lot of information about the random Hamiltonian $H_{\lambda}$. Note for example that under the hypothesis of Theorem 1 of [3] the authors showed that the integrated density of states $N_{\lambda}(E)$ is absolutely continuous and

$$
\frac{d}{d E} N_{\lambda}(E)=\frac{i}{\pi} \operatorname{Im} \lim _{\eta \downarrow 0} \frac{1}{\ell} \sum_{j=1}^{\ell} J_{\lambda}^{j}(E+i \eta),
$$

while similar results are true for the summation of the Lyaponov exponents (see [13]).

One striking and important aspect of the behavior of the $\lim _{\eta \downarrow 0} J_{\lambda}^{j}(E+i \eta), j=1$, $\ldots, \ell$ as $\lambda$ approaches zero is the apparent sensitivity of its asymptotic expansion on the location of energy $E$ in relation to the spectrum of the free Hamiltonian $H_{0}$. This was first discovered by Kappus and Wegner [4] who showed that in one dimension if $\mu$ has mean zero the leading coefficient of the straighforward perturbation expansion in $\lambda$ proposed by Thouless [5] was inadequate if $E=0$. Derrida and Gardner [6] found that the same phenomenon occurs for the next to leading coefficient in the case $E= \pm \frac{1}{2}$ and actually conjectured that similar "anomalies" occur for all energies of the form $E=\cos \pi p / q$ with $p<q$ relatively prime. This was shown by Bovier and Klein [7].

The significance of the special energies mentioned above lies in the fact that for these values the free Schrödinger propagator is cyclic (see $[6,7])$. In fact the presence of this cyclicity is directly responsible for the failure of straightforward perturbation expansions while it is exactly the same property that makes the modified ones proposed in $[6,7,9]$ amenable to rigorous treatment $[8,9]$. It is worth noting however at this point that the nature of these modified expansions in the case where $\mu$ has mean zero $[4,6,7,8]$ is quite different than the one discovered in [9] when the mean is different from zero and that one has to abandon in the latter case the traditional perturbation techniques for isolated eigenvalues in favor of a more general approach [9] where the harmonic analysis becomes much simpler than the one used in [8].

Despite the fact that in one dimension the asymptotic behavior of $H_{\lambda}$ is completely understood, at least for the special energies mentioned above, very little has been done for the case of one dimensional strips. In fact the only article the author is aware of in that direction is a paper by Derrida and Zanon [10] where a weak disorder expansion is derived for the Lyaponov exponents of the product of random matrices when the unperturbed matrices have two degenerate eigenvalues.

In this article we study the asymptotic behavior of $\lim _{\eta \downarrow 0} J_{\lambda}^{j}(E+i \eta), j=1, \ldots, \ell$ as $\lambda$ approaches zero on a strip of size $\ell$. To make the harmonic analysis more tractable we will use the approach of [9] and we will assume that the mean of $\mu$ is different than zero. We also restrict ourselves to the set of energies for which the free Schrödinger propagator becomes cyclic (see Corollary 3.4) and which we now introduce through the following definition. 
Definition 1.1. We will say that an energy $E$ is $\ell$-regular if and only if $|E|<1+\cos \frac{\pi \ell}{\ell+1}$ and it satisfies the equations

$$
E+\cos \frac{\pi k}{\ell+1}=\cos \frac{\pi p_{k}}{q_{k}}, \quad k=1, \ldots, \ell
$$

for some sequence of pairs of integers $\left(p_{k}, q_{k}\right)_{k=1, \ldots, \ell}$ which can be assumed to be unique by requiring that $p_{k}$ is relatively prime to $q_{k}$ for all $k=1,2, \ldots, \ell$.

Trivially zero is $\ell$-regular for all $\ell=1,2, \ldots$ Moreover, one can easily verify that the set of $\ell$-regular energies is dense in

$$
\left(-\left(1+\cos \frac{\pi k}{\ell+1}\right), 1+\cos \frac{\pi k}{\ell+1}\right) .
$$

Our main theorem is:

Theorem 1.2. Let $\mu$ be such that its characteristic function $h$ is infinitely many times differentiable on $(0,+\infty)$ with $h^{(i)}(t)=O\left[\left(1+t^{2}\right)^{-\alpha / 2}\right]$ for all $i=0,1,2, \ldots$ and some $\alpha>0$. If the first and second moments of $\mu$ exist and they are both not equal to zero, then for every $\ell$-regular energy $E$ the function $\mathbb{R} \ni \lambda \mapsto \lim _{\eta \downarrow 0} J_{\lambda}^{j}(E+i \eta) \in \mathbb{C}$ has an asymptotic expansion to any order at $\lambda=0$ for all $j=1, \ldots, \ell$.

We finish this section with a few words about the strategy of our proof as well as the organization of the present article.

We first use the supersymmetric replica trick (see [11]) to express $\lim _{\eta \downarrow 0} J_{\lambda}^{j}(E+i \eta)$ in terms of the unique eigenvector, corresponding to the eigenvalue one, of a bounded operator defined on an appropriate Hilbert space. Then we find explicitly the set of equations the coefficients of the formal perturbation expansion of the eigenvector mentioned above would have to satisfy. Finally we prove that these equations have a unique well defined solution which defines a series that is actually asymptotic.

The next section is devoted to the development of the formalism which permits us to implement the first step of the above outline. In Sect. III we compute the perturbation expansion and we prove, up to a key technical estimate, that it is asymptotic and in the last section prove this crucial technical estimate.

\section{The Supersymmetric Transfer Matrix}

In this section we introduce a supersymmetric formalism similar to the one used by Klein and Speis in [11] and explain how one can use it to study the boundary value of $J_{\lambda}^{i}(E+i \eta), j=1, \ldots, \ell$ as $\eta$ approaches zero.

For the benefit of those who are unfamiliar with this framework it is worth mentioning that this formalism is essentially harmonic analysis of multicomponent complex valued functions whose components satisfy certain differential equations that can be canonically described through an appropriate superposition of commuting and anticommuting variables (super variables).

Even though one could, on a purely technical level, introduce all the necessary structure without the use of Grassman-algebras (anticommuting variables) it is our experience that such an approach lacks any kind of intuition or insight and we will not use it here. We also would like to point out that despite the fact that our 
definitions look different from the ones used in [11] the proofs of our propositions follow along the same lines as the ones in [11] and they will be omitted.

We start with the following definitions:

Definition 2.1. Let $\ell$ be a positive integer. Let $\Lambda\left(\mathbb{R}^{2 \ell}\right)$ denote the Grassmans algebra over $\mathbb{R}^{2 \ell}$ and let $\Lambda^{1}\left(\mathbb{R}^{2 \ell}\right)$ be the vector space of 1 -forms in $\Lambda\left(\mathbb{R}^{2 \ell}\right)$. The superspace $\mathscr{L}_{\ell}$ is defined to be the set of all $n$-tuples $\Phi=\left(\Phi_{1}, \ldots, \Phi_{\ell}\right)$, where

$$
\begin{array}{ll}
\Phi_{i}=\left(\varphi_{i}, \bar{\psi}_{i}, \psi_{i}\right), & i=1, \ldots, \ell, \\
\varphi_{i} \in \mathbb{R}^{2} & , \quad i=1, \ldots, \ell,
\end{array}
$$

and $\left\{\bar{\psi}_{i}, \psi_{i}\right\}_{i=1, \ldots, \ell}$ is a family of 1 -forms whose non-zero elements form a linearly independent set in $A^{1}\left(\mathbb{R}^{2 t}\right)$.

Let $\mathcal{O}_{\ell}$ denote the set of strictly increasing finite sequences indexed by $\{1, \ldots, \ell\}$ and taking values in $\{1, \ldots, 2 \ell\}$. We shall make use of the forms $\left\{\Psi_{a}\right\}_{a \in \mathcal{O} \ell} \in \Lambda\left(\mathbb{R}^{2 \ell}\right)$, where for $a=\left\{i_{1}, \ldots, i_{\ell}\right\} \subset\{1,2, \ldots, 2 \ell\} \Psi_{a}$ is defined to be $\frac{1}{(2 i)^{|a|}(|a| !)}$ times the formal determinant at the $\ell \times \ell$ matrix whose rows are the $i_{1}^{\text {th }}, \ldots, i_{\ell-1}^{\text {th }}$ and $i_{\ell}^{\text {th }}$ rows of the matrix

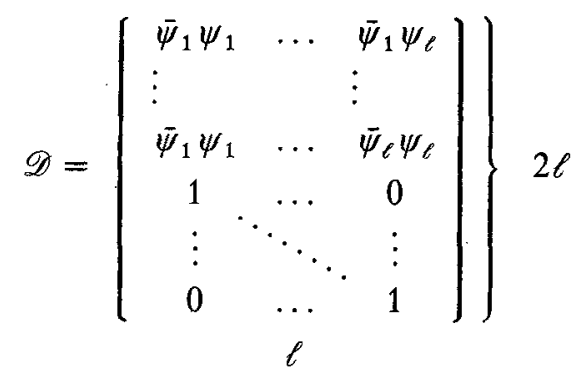

and where $|a|$ denotes the number of all the $i_{k}$ 's for which $i_{k} \leqq \ell, k=1, \ldots, \ell$.

Definition 2.2. Let $\ell$ be a positive integer. A super function $F$ is understood to be a function $F: \mathscr{L}_{\ell} \rightarrow \Lambda\left(\mathbb{R}^{2 \ell}\right)$ of the form

$$
F(\Phi)=\sum_{a \in \mathscr{O}_{\ell}} F_{a}(\varphi) \Psi_{a}
$$

where $\Phi=\left(\Phi_{1}, \ldots, \Phi_{\ell}\right), \Phi_{i}=\left(\varphi_{i}, \bar{\psi}_{i}, \psi_{i}\right), i=1, \ldots, \ell, \varphi=\left(\varphi_{1}, \ldots, \varphi_{\ell}\right)$ and $\left\{F_{a}\right\}_{a \in \mathcal{O}_{\ell}}$ is a family of complex valued functions defined on $\mathbb{R}^{2 \ell}$.

We will say that a superfunction $F$ is in $C^{k}\left(\mathscr{L}_{\ell}\right)$ if and only if $F_{a}$ is of class $C^{k}$ on $\mathbb{R}^{2 \ell}$ for all $a \in \mathcal{O}_{\ell}$.

Definition 2.3. Let $\ell$ be a positive integer. We defined the integration over the super space $\mathscr{L}_{\ell}$ by the formula

$$
\int F(\Phi) d \Phi=\frac{(-1)^{\ell}}{\pi^{\ell}} \int_{\mathbb{R}^{2 \ell}} F_{a_{I}}\left(\varphi_{1}, \ldots, \varphi_{\ell}\right) \prod_{i=1}^{\ell} d^{2} \varphi_{i},
$$

where $a_{I} \in \mathcal{O}_{\ell}$ is the identity sequence $a_{I}(i)=i, i=1, \ldots, \ell$.

We will say that a superfunction $F$ is in $L^{1}\left(\mathscr{L}_{\ell}\right)$ if $F_{a} \in L^{1}\left(\mathbb{R}^{2 \ell}\right)$ for all $a \in \mathcal{O}_{\ell}$. 
Definition 2.4. Let $\Phi, \Phi^{\prime} \in \mathscr{L}_{\ell}$. We define the inner product between $\Phi$ and $\Phi^{\prime}$ through the formula

$$
\left\langle\Phi, \Phi^{\prime}\right\rangle=\sum_{i=1}^{\ell} \Phi_{i} \cdot \Phi_{i}^{\prime}, \quad \Phi=\left(\Phi_{1}, \ldots, \Phi_{\ell}\right), \quad \Phi^{\prime}=\left(\Phi_{1}^{\prime}, \ldots, \Phi_{\ell}^{\prime}\right) .
$$

where $\Phi_{i} \cdot \Phi_{i}^{\prime}=\varphi_{i} \cdot \varphi_{i}^{\prime}+\frac{1}{2}\left(\bar{\psi}_{i} \psi_{i}^{\prime}+\bar{\psi}_{i}^{\prime} \psi_{i}\right), i=1, \ldots, \ell$.

Let $K_{\ell}$ be the cone at the positive semidefinite $\ell \times \ell$ matrices and let $f$ be a complex valued function which is of class $C^{1}$ on $K_{\ell}$. We associate with $f$ the superfunction $F$ defined by

$$
F(\Phi)=\sum_{a \in \mathbb{Q}_{b}} \partial_{a} f\left(A_{\varphi}\right) \Psi_{a},
$$

where $\partial_{a}, a=\left\{i_{1}, i_{2}, \ldots, i_{\ell}\right\}$ is the partial differential operator defined by the formal determinant of an $\ell \times \ell$ matrix whose rows are the $i_{1}, \ldots, i_{\ell}$ 'th rows of the matrix

$$
\left.\left\{\begin{array}{ccc}
2 i \partial_{11} & \ldots & i \partial_{1 \ell} \\
\vdots & & \vdots \\
i \partial_{\ell 1} & \cdots & 2 i \partial_{\ell \ell} \\
1 & \ldots & 0 \\
\vdots & \ddots & \vdots \\
0 & \cdots & 1
\end{array}\right]\right\} 2 \ell
$$

$\partial_{i j}, i, j=1, \ldots, \ell$ denotes the operator differentiation with respect to the variable that corresponds to the $i, j$ entry and $A_{\varphi}$ is an $\ell \times \ell$ matrix defined by $\left(A_{\varphi}\right)_{i j}=\varphi_{i} \cdot \varphi_{j}, i, j=1,2, \ldots, \ell$.

It is worth noting that if we set $A_{\Phi}$ to be the $\ell \times \ell$ super matrix defined by $\left(A_{\Phi}\right)_{i j}=\Phi_{i} \cdot \Phi_{j}, i, j=1,2, \ldots, \ell$ we can rewrite (2.2) as

$$
F(\Phi)=f\left(A_{\Phi}\right),
$$

where $f\left(A_{\Phi}\right)$ is defined to be equal to its formal Taylor series, around the point $A_{\varphi}$ which coincides with the right-hand side of (2.2).

Let $\mathscr{P}\left(K_{\ell}\right)$ be the usual Schwartz space over $K_{\ell}$. We shall denote by $\mathscr{S}\left(\mathscr{L}_{\ell}\right)$ the set of all superfunctions of the form $F(\Phi)=f\left(A_{\Phi}\right)$ with $f \in \mathscr{S}\left(K_{\ell}\right)$.

An important notion of the formalism developed so far is the one of supersymmetries. These are transformations of the superspace $\mathscr{L}_{\ell}$ and are defined by

where

$$
U_{b, \bar{b}, \xi}=\left(U_{b, \bar{b}, \xi} \Phi_{1}, \ldots, U_{b, \bar{b}, \xi} \Phi_{\ell}\right)
$$

$$
U_{b, \bar{b}, \xi}\left(\Phi_{i}\right)=\left(\varphi_{i}+2 \bar{b} \xi \psi_{i}+2 b \xi \bar{\psi}_{i}, \bar{\psi}_{i}-4 \bar{b} \varphi_{i} \xi, \psi_{i}+4 b \varphi_{i} \xi\right)
$$

$b, \bar{b}$ are arbitrary constants and $\xi$ is a 1 -form independent from $\left\{\bar{\psi}_{i}, \psi_{i}\right\}_{i=1}, \ldots . \ell$. One can define the action of $U_{b, \bar{b}, \xi}$ on a superfunction $F$ in a natural way through (see also [14])

$$
\left(U_{b, b, \xi} F\right)(\Phi)=F\left(U_{b, b, \xi} \Phi\right),
$$

where it is implicitly understood that $F_{a}\left(\varphi_{1}+2 \bar{b} \xi \psi_{1}+2 b \xi \bar{\psi}_{1}, \ldots, \varphi_{\ell}+2 b \xi \bar{\psi}_{\ell}\right.$ $\left.+2 \bar{b} \xi \psi_{1}\right)$ is defined to be equal to its formal Taylor expansion around $\left(\varphi_{1}, \ldots, \varphi_{\ell}\right)$ for all $a \in \mathcal{O}_{\ell}$. 
Definition 2.5. Let $\ell$ be a positive integer. We will say that a superfunction $F: \mathscr{L}_{\ell} \rightarrow \Lambda\left(\mathbb{R}^{2 \ell}\right)$ is supersymmetric it if is left invariant by all supersymmetries of $\mathscr{L}_{\ell}$.

Theorem 2.5. Let $\ell$ be a positive integer and let $F: \mathscr{L}_{\ell} \rightarrow \Lambda\left(\mathbb{R}^{2 \ell}\right)$ be a superfunction in $L^{1}\left(\mathscr{L}_{\ell}\right) \cap C^{\ell}\left(\mathscr{L}_{\ell}\right)$. Then the following are true:

1) $\int\left(U_{b, b, \xi} F\right)(\Phi) d \Phi=\int F(\Phi) d \Phi$.

2) If $F$ is supersymmetric then

$$
\int F(\Phi) d \Phi=F(0) .
$$

Remark 2.6. One can easily check that superfunctions of the form defined in (2.3) are always supersymmetric.

Let $A$ be an $2 \ell \times 2 \ell$ matrix. We define the action of $A$ on superfunctions $F$ which we shall denote by $A F$ through

$$
(A(F))_{a}=\sum_{b \in \mathcal{O}_{\ell}} \operatorname{det}\left(A_{a}^{b}\right) F_{b}, \quad a \in \mathcal{O}_{\ell},
$$

where for $a=\left\{i_{1}, \ldots, i_{\ell}\right\}$ and $b=\left\{j_{1}, \ldots, j_{\ell}\right\} A_{a}^{b}$ denotes the $\ell \times \ell$ matrix defined by $\left(A_{a}^{b}\right)_{k, h}=A_{i_{k}, j_{h}}, k, h=1,2, \ldots, \ell$. It is easy to check that for $A, B 2 \ell \times 2 \ell$ matrices

$$
A(B(F))=(A B)(F) .
$$

We now introduce the Hilbert spaces mentioned in the previous section. Let $E \in \mathbb{R}$ and $A_{E}$ be the $2 \ell \times 2 \ell$ symmetric matrix defined by

$$
A_{E}=\left(\begin{array}{cc}
I_{\ell} & \mathscr{E}_{E} \\
\mathscr{E}_{E} & I_{\ell}
\end{array}\right)
$$

where $I_{\ell}$ is the $\ell \times \ell$ identity matrix and

$$
\left.\mathscr{E}_{E}=\left\{\begin{array}{cccc} 
& \ell & & \\
E & 1 / 2 & \\
1 / 2 & \ddots & 0 \\
1 / 2 & \ddots & \ddots \\
& \ddots & \ddots & 1 / 2 \\
0 & & 1 / 2 & E
\end{array}\right)\right\} \ell
$$
One can easily verify by diagonalizing $\mathscr{E}_{E}$ that if $|E|<1+\cos \frac{\pi \ell}{\ell+1} A_{E}$ is positive
definite.

Let $Q_{i}, i=1,2$ denote the vector valued operators, acting on smooth complex valued functions which are defined on $\mathbb{R}^{2 \ell}$ by

$$
\begin{gathered}
Q_{i}=\left\{\left[Q_{i}\right]_{1} \cdots\left[Q_{i}\right]_{2 \ell}\right), \quad i=1,2, \\
{\left[Q_{i}\right]_{k}=\frac{i \partial}{\partial \varphi_{k, i}}, \quad k=1, \ldots, \ell,} \\
{\left[Q_{i}\right]_{k}=M \varphi_{k-\ell, i}, \quad k=\ell+1, \ldots, 2 \ell,}
\end{gathered}
$$


where $M \varphi_{k-\ell, i}$ stands for the operator multiplication by the variable $\varphi_{k-\ell, i}$ and we have used the convention that a vector $\varphi \in \mathbb{R}^{2 \ell}$ can be written as $\varphi=\left(\left(\varphi_{11}, \varphi_{12}\right)\right.$, $\left.\ldots,\left(\varphi_{\ell 1}, \varphi_{\ell 2}\right)\right)$.

Let $\mathscr{S}\left(\mathbb{R}^{2 \ell}\right)$ denote the usual Schwartz space over $\mathbb{R}^{2 \ell}$. For every energy $E$ with $|E|<1+\cos \frac{\pi \ell}{\ell+1}$ we introduce a sequence of positive norms $\|\cdot\|_{n}^{E}$, $n=1,2,3, \ldots$ on $\mathscr{S}\left(\mathbb{R}^{2 \ell}\right)$ through the following equations:

$$
\left(\|f\|_{n}^{E}\right)^{2}=\sum_{k_{1}, \ldots, k_{n}=1}^{2 \ell} \sum_{i_{1}, \ldots, i_{n}=1}^{2}\left\|\left(\prod_{m=1}^{n-1}\left[A_{E}^{1 / 2}\left(Q_{i_{m}}\right)\right]_{k_{m}}\right)(f)\right\|_{L^{2}\left(\mathbb{R}^{2 \ell}, d^{2} \varphi\right)}^{2} .
$$

Definition 2.7. Let $n$ and $\ell$ be positive integers and let $E$ be a real number such that $|E|<1+\cos \frac{\pi \ell}{\ell+1}$. We define the supersymmetric Hilbert space $H_{n, E}$ to be the completion of the subspace of $\mathscr{S}\left(\mathscr{L}_{\ell}\right)$ under the norm $\|\cdot\|_{n, E}$, where

$$
\left(\|F\|_{n, E}\right)^{2}=\sum_{a \in \mathscr{O}_{\ell}} \sum_{m=1}^{n}\left(\left\|\left(A_{E}^{1 / 2}(F)\right)_{a}\right\|_{m}^{E}\right)^{2}+|F(0)|^{2}, \quad F \in \mathscr{S}\left(\mathscr{L}_{\ell}\right) .
$$

Let $\ell$ be a positive integer and let $I$ be a compact set of $\left(-1-\cos \frac{\pi \ell}{\ell+1}\right.$, $\left.1+\cos \frac{\pi \ell}{\ell+1}\right)$. One can easily check that

$$
C_{I}^{\prime}\|f\|_{n}^{0} \leqq\|f\|_{n}^{E} \leqq C_{I}^{\prime}\|f\|_{n}^{0}
$$

for all $f \in \mathscr{S}\left(\mathbb{R}^{2 \ell}\right)$ and $n=0,1,2, \ldots$ and that

$$
C_{I}\|F\|_{n, 0} \leqq\|F\|_{n, E} \leqq C_{I}\|F\|_{n, 0}
$$

for all $F \in \mathscr{S}\left(\mathscr{L}_{\ell}\right)$ and $n=0,1,2, \ldots$, where $C_{I}$ and $C_{I}^{\prime}$ are constants that depend only on the set $I$.

In view of relations (2.5) we can identify $H_{n, E}$ with the set of functions $F: \mathscr{L}_{\ell} \rightarrow \Lambda\left(\mathbb{R}^{2 \ell}\right)$ of the form

$$
F(\Phi)=\sum_{a \in \mathbb{O}_{\ell}} F_{a}(\varphi) \Psi_{a}
$$

where now $\left\{F_{a}\right\}_{a \in \mathcal{O},}$ are in general elements of the completion of $\mathscr{S}\left(\mathbb{R}^{2 \ell}\right)$ under $\|\cdot\|_{n}^{0}$.

Let $\mathscr{F}$ denote the usual Fourier transform on $\mathbb{R}^{2 \ell}$. We will be making use of an operator on $L^{1}\left(\mathscr{L}_{\ell}\right)$ which we will denote by the same letter $\mathscr{F}$ and is defined by

$$
(\mathscr{F} F)_{a}=\mathscr{F}\left(F_{a}\right), \quad F \in L^{1}\left(\mathscr{L}_{\ell}\right) .
$$

Let $A$ be an $\ell \times \ell$ matrix. We shall also make use of the operator $b_{A}$ which acts on superfunctions and is defined by

$$
\left(b_{A} F\right)_{a}(\varphi)=e^{i\langle\varphi, A \varphi\rangle}\left(F_{a}\right)(\varphi), \quad \varphi \in \mathbb{R}^{2 \ell} .
$$

Let $B$ be a $2 \ell \times 2 \ell$ matrix. For convenience we view $\left[B\left(Q_{i}\right)\right]_{k}, i=0,1$, $k=1, \ldots, 2 \ell$ as operators acting on $\mathscr{S}\left(\mathscr{L}_{\ell}\right)$ defined by

$$
\left(\left[B\left(Q_{i}\right)\right]_{k}(F)\right)_{a}=\left(\left[B\left(Q_{i}\right)\right]_{k}(F)\right)_{a}, \quad i=0,1, k=1,2, \ldots, 2 \ell .
$$

Through a straightforward computation one can easily verify that $\left[B\left(Q_{i}\right)\right]_{k}$ extends to a contraction from $H_{n+1}$ to $H_{n}$ satisfying the intertwining relations

$$
\left[B\left(Q_{i}\right)\right]_{k} b_{\mathscr{E}_{E+i \eta}} \mathscr{F}=-i b_{\mathscr{E}_{E+i n}} \mathscr{F}\left[\left(B \mathscr{C}_{E+i \eta}\right)\left(Q_{i}\right)\right]_{k}
$$


for all $i=0,1, k=1, \ldots, 2 \ell, n=1,2, \ldots$ and $\eta \geqq 0$ where we have used the natural extension

$$
\mathscr{E}_{E+i \eta}=\left(\begin{array}{ccccc}
E+i \eta & 1 / 2 & & \\
& 1 / 2 & \ddots & \ddots & 0 \\
& & \ddots & \ddots & \ddots \\
& & \ddots & \ddots & 1 / 2 \\
& 0 & \ddots & & \ddots \\
& & & 1 / 2 & E+i \eta
\end{array}\right)
$$

and where

$$
\mathscr{C}_{E+i \eta}=\left(\begin{array}{cc}
-2 i \mathscr{E}_{E+i \eta} & -i I \\
i I & 0
\end{array}\right)
$$

Remark 2.8. If $|E|<1+\cos \frac{\pi \ell}{\ell+1}$ it is not hard to verify that the norms we introduced here are equivalent to the ones Klein, Lacroix and Speis used in [11]. The reasons why we have to use these, at least at first sight, complicated norms should become clear towards the beginning of the next section.

We now proceed to explain how one can use the formalism we have developed so far to study the averaged diagonal matrix elements of the Green's kernel $J_{\lambda}^{i}(E+i \eta)$ for the Anderson Model on a one-dimensional strip. From now on and until the end of the present article $\ell$, unless otherwise stated, will denote the width of the strip $\mathscr{D}_{\ell}$.

Using the supersymmetric replica trick we can reexpress $\mathbb{E}\left\{G_{m}^{\lambda}((0, j),(0, j)\right.$, $E+i \eta)\}($ see III.1 of [11]) as

$$
\begin{aligned}
\mathbb{E}\left\{G_{m}^{\lambda}((0, j),(0, j), E+i \eta)\right\}= & i \int \psi_{j} \bar{\psi}_{j}\left[\left(B_{\lambda}(\eta) T_{E}\right)^{m}\left(\beta_{E, \lambda}(\eta)\right)\right] \\
& \times\left[T\left(B_{\lambda}(\eta) T_{E}\right)^{m}\left(\beta_{E, \lambda}(\eta)\right)\right](\Phi) d \Phi
\end{aligned}
$$

for all $\eta>0, m=1,2, \ldots$ and $j=0,1,2, \ldots$, where

$$
\begin{aligned}
& \left(\beta_{E, \lambda}(\eta)\right)(\Phi)=e^{i\left\langle\Phi, \mathscr{E}_{E+i \eta} \Phi\right\rangle} \prod_{i=1}^{\ell} h\left(\lambda \Phi_{i}^{2}\right), \\
& \left(T_{E}(F)\right)(\Phi)=e^{i\left\langle\Phi, \mathscr{E}_{E} \Phi\right\rangle} \int e^{i\left\langle\Phi^{\prime}, \Phi\right\rangle} F(\Phi) d \Phi, \quad F \in L^{1}\left(\mathscr{L}_{\ell}\right), \\
& (T(F))(\Phi)=\int e^{i\left\langle\Phi, \Phi^{\prime}\right\rangle} F(\Phi) d \Phi, \quad F \in L^{1}\left(\mathscr{L}_{\ell}\right) .
\end{aligned}
$$

$B_{\lambda}(\eta)$ stands for the operator multiplication by $\prod_{i=1}^{\ell} h\left(\lambda \Phi_{i}^{2}\right) e^{-\eta \Phi_{i}^{2}}$ and we have used the notation $\Phi_{i}^{2}=\left\langle\Phi_{i}, \Phi_{i}\right\rangle, \varphi_{i}^{2}=\varphi_{i} \cdot \varphi_{i}, i=1, \ldots, \ell$.

The properties of the operator $B_{\lambda}(\eta) T_{E}$ are well understood for $\lambda \neq 0$ and we summarize them in the following theorem.

Theorem 2.9. Let $\eta$ be a positive integer and let the distribution of the single sided potential $\mu$ be such that its characteristic function together with all its derivatives up to order $n+1$ are continuous on $(0,+\infty)$ with some decay at infinity. Then the following are true:

1) $B_{\lambda}(\eta) T_{E}$ extends to a bounded operator on $H_{n, 0}$ for all $E \in \mathbb{R}$ and $\eta \geqq 0$ with the operator valued function $\{E+i \eta \in C: \eta \geqq 0\} \ni E+i \eta \mapsto B_{\lambda}(\eta) T_{E} \in H_{\eta, 0}$ being norm continuous. 
2) The spectrum of $B_{\lambda}(\eta) T_{E}$ is discrete, zero is the only possible accumulation point and 1 is a simple eigenvalue while the rest of the spectrum lies inside a disk centered at 0 and of radius strictly less than one for all $\lambda \neq 0, \eta \geqq 0$ and $E \in \mathbb{R}$.

Proof. See [11].

In view of the previous theorem we can now let $m$ go to $+\infty$ and $\eta$ approach zero in (2.7) to obtain

$$
\lim _{\eta \downarrow 0} J_{\lambda}^{j}(E+i \eta)=i \int \psi_{j} \bar{\psi}_{j} \xi_{E, \lambda}(\Phi)\left[\left(T\left(\xi_{E, \lambda}\right)\right)(\Phi)\right] d \Phi, \quad j=1, \ldots, \ell,
$$

where $\xi_{E, \lambda}$ is the unique solution of the equation

$$
\left(B_{\lambda}(0) T_{E}\right) \xi_{\lambda, E}=\xi_{\lambda, E} \text {. }
$$

The remaining two sections are devoted to the study of the vector valued function

$$
\mathbb{R} \ni \lambda \mapsto \xi_{\lambda, E} \in H_{E, n}
$$

for $\lambda$ close to zero and $E \ell$-regular.

\section{The Asymptotic Expansion}

In this section we show that under the hypothesis of Theorem 1.2 the function $\mathbb{R} \in \lambda \mapsto \xi_{\lambda, E} \in H_{n, E}$ has an asymptotic expansion to any order in the disorder parameter $\lambda$. We will assume the following technical result, the proof of which we postpone until the last section.

Lemma 3.1. Under the hypothesis of Theorem 1.2 for every $n$ strictly positive integer there exists a constant $M$ independent of $\lambda$ such that

for all $\lambda \neq 0$.

$$
\left\|\xi_{\lambda, E}\right\|_{n+1, E} \leqq M\left\|\xi_{\lambda, E}\right\|_{n, E}
$$

Through an explicit computation and Proposition IV.1.4 of [11] one could easily verify that

$$
\begin{array}{lrl}
T_{E}(F)=\left(\mathscr{C}_{E} b_{\mathscr{E}_{E}} \mathscr{F}\right)(F), & E \in \mathbb{R}, & F \in L^{1}\left(\mathscr{L}_{\ell}\right), \\
T(F)=\left(i J_{\ell} \mathscr{F}\right)(F) & , & F \in L^{1}\left(\mathscr{L}_{\ell}\right),
\end{array}
$$

and

$$
\left(B_{\lambda}(0)\right)(F)=\left(\mathbb{E}_{U}\left\{V_{\lambda} b_{\lambda U}\right\}^{\prime}(F), \quad F\right. \text { superfunction, }
$$

where

$$
J_{\ell}=\left(\begin{array}{cc}
0 & -I_{\ell} \\
I_{\ell} & 0
\end{array}\right) .
$$

$I_{\ell}$ is $\ell \times \ell$ identity matrix,

$$
V_{\lambda}=\left(\begin{array}{cc}
I & 2 \lambda U \\
0 & I
\end{array}\right) .
$$

$U$ stands for -1 times the $\ell \times \ell$ diagonal random matrix whose elements are $\ell$ independent copies of a random variable whose probability distribution is $\mu$ and $\mathbb{E}_{U}$ denotes the expectation over the product space of these variables.

We are now ready to prove a proposition which provides the insight behind Definition 2.7. 

Proposition 3.2. Let $\ell$ be a positive integer. If $|E|<\cos \frac{\pi \ell}{\ell+1}+1$, then $T_{E}$ is an
isometry on $H_{n, E}$ for all $n=0,1,2 \ldots$.

Proof. One can easily check that if $B_{1}, \ldots, B_{n}$ are square $2 \ell \times 2 \ell$ matrices and $B_{1}^{*}, \ldots, B_{n}^{*}$ are their corresponding adjoints, then

$$
\begin{aligned}
& \sum_{a \in \mathcal{O}_{\ell}} \sum_{k_{1}, \ldots, k_{n}=1}^{2 \ell} \sum_{i_{1}, \ldots, i_{n}=1}^{2} \overline{\left[\left(\prod_{m=1}^{n-1}\left[\left(B_{m} A\right)\left(Q_{i_{m}}\right)\right]_{k_{m}}\right)\left(\left(B_{n} A\right)(G)\right)_{a}\right]} \\
& \times\left[\left(\prod_{m=1}^{n-1}\left[A\left(Q_{i_{m}}\right)\right]_{k_{m}}\right)\right. \\
& =\sum_{a \in \mathcal{O}_{\ell}} \sum_{k_{1}, \ldots, k_{n}=1}^{2 \ell} \sum_{i_{1}, \ldots, i_{n}=1}^{2} \overline{\left[\left(\prod_{m=1}^{n-1}\left[A\left(Q_{i_{m}}\right)\right]_{k_{m}}\right)(A(G))_{a}\right]} \\
& \times\left[\left(\prod_{m=1}^{n-1}\left[\left(B_{m}^{*} A\right)\left(Q_{i_{m}}\right)\right]_{k_{m}}\right)\right.
\end{aligned}
$$

for all $A$ square $2 \ell \times 2 \ell$ matrices and all $F, G \in \mathscr{S}\left(\mathscr{L}_{\ell}\right)$. On the other hand, since $\mathscr{C}_{E}$ commutes with $b_{\mathscr{E}_{E}}$ and $\mathscr{F}$ we get from relations (3.2) and (2.6) that if $F \in \mathscr{S}\left(\mathscr{L}_{\ell}\right)$, then

$\left(\left\|T_{E}(F)\right\|_{n}^{E}\right)^{2}=$

$$
\sum_{a \in \mathscr{O}_{\ell} k_{1}, \ldots, k_{n}=1} \sum_{i_{1}, \ldots, i_{n}=1}^{2 \ell}\left\|\left(\prod_{m=1}^{n-1}\left[A_{E}^{1 / 2} \mathscr{C}_{E} Q_{i_{m}}\right]_{k_{m}}\right)\left(A_{E}^{1 / 2} \mathscr{C}_{E} F\right)_{a}\right\|_{L^{2}\left(\mathbb{R}^{2} \ell, d^{2} \ell \varphi\right)}^{2} .
$$

The result now follows from Eq. (3.3), Definition 2.1 and the fact that $\mathscr{C}_{E}^{*} A_{E} \mathscr{C}_{E}=A_{E}$.

For the study of the asymptotic expansion of $\xi_{\lambda, E}$ we will need the following technical results.

Lemma 3.3. Let $\lambda \geqq 0$ and let $C_{\lambda}$ denote the operator $B_{\lambda}(0)$ efined through $E q .3 .1$ for the case where $\mu$ is the Cauchy distribution with parameter one (i.e. $h(r)=e^{-|r|}$ ). Then

$$
\begin{aligned}
& {\left[\left(C_{\lambda} T_{E}\right)^{k}(F)\right](\Phi)=e^{i\left\langle\Phi,\left(\mathscr{E}_{E}+i \lambda I_{\ell}\right) \Phi\right\rangle+\frac{1}{4} i\left\langle\Phi, 2 P_{k-1} P_{k}^{-1} \Phi\right\rangle}} \\
& \quad \times \int e^{\frac{1}{4} i\left\langle\Phi, 2 P_{1} P_{\bar{k}}^{-1} \Phi^{\prime}\right\rangle+\frac{1}{4} i\left\langle\Phi^{\prime}, 2 P_{1} P_{\bar{k}}^{1} \Phi\right\rangle} e^{\frac{1}{4} i\left\langle\Phi^{\prime}, 2 P_{k-1} P_{\bar{k}}^{-1} \Phi^{\prime}\right\rangle} F\left(\Phi^{\prime}\right) d \Phi^{\prime}
\end{aligned}
$$

for all $k=1,2, \ldots$ and $F \in L^{1}\left(\mathscr{L}_{\ell}\right)$, where $\left\{P_{k}\right\}_{k \in \mathbb{N}}$ is a sequence of $\ell \times \ell$ square matrices defined by

$$
\begin{gathered}
\left(\begin{array}{l}
P_{k} \\
P_{k-1}
\end{array}\right)=\left(\begin{array}{cc}
-2\left(\mathscr{E}_{E}+i \lambda I_{\ell}\right) & -I_{\ell} \\
I_{\ell} & 0
\end{array}\right) \cdots\left(\begin{array}{cc}
-2\left(\mathscr{E}_{E}+i \lambda I_{\ell}\right) & -I_{\ell} \\
I_{\ell} & 0
\end{array}\right)\left(\begin{array}{l}
I_{\ell} \\
0
\end{array}\right) \\
k-1 \text { times }
\end{gathered}
$$

and $I_{\ell}$ stands for the $\ell \times \ell$ identity matrix.

Proof. The proof is an immediate consequence of III.2.3 and III.2.4 of [3] and III.2.3 of [11]. 
Corollary 3.4. Let $E$ be $\ell$-regular and let $q_{E}$ be the least common multiple of $q_{1}, \ldots, q_{\ell}$, where $q_{1}, \ldots, q_{\ell}$ are the integer phases that appear in Definition 1.1. Then $\left(T_{E}\right)^{q_{E}}=I$ on $H_{n, E}$ for all $n=0,1, \ldots$.

Proof. Let $\lambda$ be a positive number. One can easily check (see also [9]) that the matrices $P_{k}, k=0,1, \ldots$ which appear in the exponent of the right-hand side of Eq. (3.5) can be explicitly computed and are given by

$$
P_{k+1}=\left(\left(\Gamma_{E, \lambda}\right)^{2}-I_{\ell}\right)^{-1}\left[\left(\Gamma_{E, \lambda}\right)^{k+2}-\left[\left(\Gamma_{E, \lambda}\right)^{k}\right]^{-1}\right], \quad k=0,1, \ldots,
$$

where $\Gamma_{E, \lambda}=-\left(\mathscr{E}_{E}+i \lambda I_{\ell}\right)-\sqrt{\left(\mathscr{E}_{E}+i \lambda I_{\ell}\right)^{2}-I_{\ell}}$. However since $E+\cos \frac{\pi k}{k+1}$, $k=1, \ldots, \ell$ are the eigenvalues of $\mathscr{E}_{E}$ we conclude that $\left(\Gamma_{E, 0}\right)^{q_{E}}=I$. Thus by taking the limit as $\lambda$ approaches zero from the right we obtain from relations (3.5) that

$$
\left(T_{E}\right)^{q_{E}+1}(F)=T_{E}(F), \quad F \in L^{1}\left(\mathscr{L}_{\ell}\right) .
$$

Since $T_{E}$ is an isometry on $H_{n, E^{\prime}} n=0,1, \ldots$ the result follows.

Corollary 3.5. Let $E$ be $\ell$-regular and let $n$ be a positive integer.

1) $\|\left[\left(C_{\lambda} T_{E}\right)^{k}(F)\left\|_{n, E} \leqq e^{C \lambda k}\right\| F \|_{n, E}\right.$

for all $F \in H_{n, E^{\prime}} k=0,1, \ldots$ and $\lambda \geqq 0$, where $C$ is a positive constant that depends only on $n$.

2) Let $H_{n, E}^{0}=\left\{F \in H_{n, E}: F(0)=0\right\}$. Then

$$
\left\|\left(C_{\lambda} T_{E}\right)^{k}(F)\right\|_{n, E} \leqq M e^{-C \lambda k}\|F\|_{n, E}
$$

for all $F \in H_{n, E}^{0}, k=0,1,2, \ldots$ and $0 \leqq \lambda \leqq 1$, where as before $M$ and $C$ are two positive constants that depend on $n$.

Proof.

1) It is enough of course to show the inequality for $k=1$. In this case it is easy to see that if $n$ is a positive integer and $a \in \mathcal{O}_{\ell}$ we can conclude from Eq. (2.6) that

$$
\begin{aligned}
& \left(\left\|\left(\left(C_{\lambda} T_{E}\right)(F)\right)_{a}\right\|_{n}^{E}\right)^{2} \leqq \\
& \sum_{k_{1}, \ldots, k_{n}=1}^{2 \ell} \sum_{i_{1}, \ldots, i_{n}=1}^{2}\left\|\left(\prod_{m=1}^{n-1}\left[\left(A_{E+i \lambda}^{1 / 2} \mathscr{C}_{E+i \lambda}\right)\left(Q_{i_{m}}\right)\right]_{k_{m}}\right)\left(\left(A_{E}^{1 / 2} \mathscr{C}_{E+i \lambda}\right)(F)\right)_{a}\right\|_{L^{2}\left(\mathbb{R}^{2}, \mathrm{~d}^{2} \varphi\right)}^{2}
\end{aligned}
$$

for all $\lambda \geqq 0$.

However if $0 \leqq \lambda<1$,

$$
\mathscr{C}_{E+i \lambda}^{*} A_{E} \mathscr{C}_{E+i \lambda}=A_{E}+\lambda G
$$

where $G$ is $2 \ell \times 2 \ell$ square matrix whose norm is bounded independently of $\lambda$. Thus the proof of (1) follows from relations (3.3) and Definition 2.7.

2) If $|E|<\cos \frac{\pi \ell}{\ell+1}$, then by diagonalizing the matrix $\Gamma_{E, \lambda}$ we get (see also Proposition 4.2 of [9]) that

$$
e^{d_{1} \lambda} \leqq\left\|\Gamma_{E, \lambda}\right\| \leqq e^{d_{2} \lambda}
$$


for all $\lambda \geqq 0$, where $d_{1}, d_{2}$ are two positive constants that depend only on $E$. Thus we conclude from Eq. (3.7) that there exists constants $C_{0}, M, C>0$ such that if $k_{\lambda} \geqq C_{0}$, then

and

$$
\begin{gathered}
\left\|2 P_{1} P_{k}^{-1}\right\| \leqq M e^{-C k \lambda} \\
\left\|2 P_{k-1} P_{k}^{-1}\right\| \leqq M
\end{gathered}
$$

$$
\operatorname{Im} 2 P_{k-1} P_{k}^{-1} \geqq C I_{\ell} .
$$

Let $F$ be an element of $H_{n, E}^{0}$. Using Theorem 2.5 we can rewrite (3.5) as

$$
\begin{aligned}
& {\left[\left(C_{\lambda} T_{E}\right)^{k}(F)\right](\Phi)=e^{i\left\langle\Phi,\left(\mathscr{E}_{E}+i \lambda I_{\ell}\right) \Phi\right\rangle+1 i\left\langle\Phi, 2 P_{k-1} P_{\bar{k}}-1 \Phi\right\rangle}}
\end{aligned}
$$

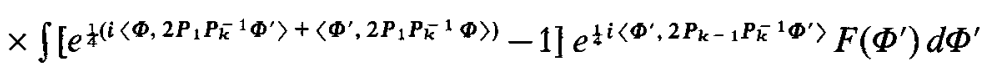

for all $k=1,2, \ldots$ and $F \in \mathscr{S}\left(\mathscr{L}_{\ell}\right)$.

Recalling the notation of Definition 2.7 and using the bounds mentioned above we conclude from Hölders inequality and a straightforward computation that if $k \lambda>C_{0}$

$$
\left(\left\|\left(C_{\lambda} T_{E}\right)^{k}(F)\right\|_{n}^{E}\right)^{2} \leqq M e^{-C \lambda k}
$$

for all $n=1,2, \ldots$.

Thus we have shown the inequality for this case where $k \lambda$ is bigger than a fixed positive number so the rest of the proof follows now from part (1).

Let $E$ be $\ell$-regular and let $q_{E}$ be the least common multiple of the integers $q_{1}, \ldots, q_{\ell}$ as discussed in Corollary 3.4. We can rewrite Eq. (2.9) as

$$
A_{\lambda, E} \xi_{\lambda, E}=0, \quad \lambda \neq 0,
$$

where $A_{\lambda, E}=\frac{\left(B_{\lambda}(0) T_{E}\right)^{q_{E}}-I}{\lambda}$.

If for a moment we assume that $\xi_{\lambda, E}$ has an asymptotic explanation on $H_{n, E}$, $n=1,2, \ldots$ of the form

$$
\xi_{\lambda, E}=\sum_{k=0}^{N} \frac{\lambda^{k}}{n !} \xi_{E}^{(k)}+o_{N}\left(|\lambda|^{N}\right), \quad N=0,1,2, \ldots,
$$

we can conclude that the coefficients $\left\{\xi_{E}^{(k)}\right\}_{k \in \mathbb{N}}$ should satisfy the equations

$$
\left(\begin{array}{l}
N \\
1
\end{array}\right) A_{0, E}\left(\xi_{E}^{(N-1)}\right)=-\sum_{k=2}^{N}\left(\begin{array}{l}
N \\
k
\end{array}\right)\left(\frac{\partial^{k}}{\partial \lambda^{k}}\left(B_{\lambda}(0) T_{E}\right)^{q_{E}}\right)_{\lambda=0}\left(\xi_{E}^{(N-k)}\right),
$$

where $A_{0, E}$ is the unbounded operator with $H_{2, E}$ as its domain and defined by

$$
A_{0, E}=\lim _{\lambda \rightarrow 0} \frac{\left(B_{\lambda}(0) T_{E}\right)^{q_{E}}-I}{\lambda}=-i \mu_{0} \sum_{k=0}^{q_{E}-1}\left(T_{E}\right)^{k} M_{\Phi}\left(T_{E}\right)^{q-k},
$$

where $M_{\Phi}$ denotes the operator multiplication by $\Phi_{1}^{2}+\ldots+\Phi_{\ell}^{2}$ and $\mu_{0}$ is the mean of the distribution $\mu$.

At this point it should be clear that even at the level of formal perturbation theory the operator $A_{0, E}$ plays an important role in the proof of the existence of an expansion of the form (3.9). Fortunately one can study the nature of its spectrum the properties of which can be described through the following theorem. 
Theorem 3.6. Let $n$ be a positive integer and let $E$ be $\ell$-regular. Then

1) $i A_{0, E}$ viewed as an unbounded operator on $H_{n, E}$ with domain $H_{n+2} \subset H_{n, E}$ is symmetric.

2) The Friedrichs extension of $-A_{0, E}^{2}$ is a positive self-adjoint operator on $H_{n, E}$ whose spectrum has the form

$$
\sigma\left(-A_{0, E}^{2}\right) \supseteq\{0\} \cup[C,+\infty],
$$

where $C$ is some strictly positive constant $C$. Moreover zero is a simple eigenvalue with eigenvector

$$
\xi_{E}^{(0)}(\Phi)=\exp \frac{-i}{2}\left\langle\Phi, \Gamma_{E, 0} \Phi\right\rangle
$$

while the rest of the spectrum is supported by the invariant subspace $H_{n, E}^{0}$.

Proof.

1) Let $F, G \in H_{n+2, E}$ and consider the equation

$$
\left\langle F,\left(T_{E+\alpha}\right)^{q_{E}}(G)\right\rangle_{n, E+\alpha}=\left\langle\left(T_{E+\alpha}^{*}\right)^{q_{E}}(F), G\right\rangle_{n, E+\alpha},
$$

where $\langle,\rangle_{n, E+\alpha}$ denotes the inner product of the Hilbert space $H_{n, E+\alpha}$ and $\alpha$ is sufficiently a small real number. Since $M_{\Phi}$ is a bounded operator from $H_{n+2, E}$ to $H_{n, E}$ we get, by differentiating both sides of the equation above with respect to $\alpha$ and setting $\alpha=0$, that

$$
\left\langle F, \frac{-1}{\mu_{0}} A_{0, E} G\right\rangle_{n, E}=\left\langle\left.\frac{\partial}{\partial \alpha}\left(T_{E+\alpha}^{*}\right)^{q_{E}}\right|_{\alpha=0}(F), G\right\rangle_{n, E} .
$$

However, since $T_{E}$ is an isometry on $H_{n, E}$ one can easily check that

$$
\left.\frac{\partial}{\partial \alpha}\left(T_{E+\alpha}^{*}\right)^{q_{E}}\right|_{\alpha=0}(F)=-i\left[\sum_{k=0}^{q_{E}-1}\left(T_{E}^{*}\right)^{q_{E-k}} M_{\Phi}\left(T_{E}^{*}\right)^{k}\right](F)=\frac{1}{\mu_{0}} A_{0, E}(F)
$$

for all $F \in H_{n+2, E}$ and the proof of 1) follows.

2) Let $F \in H_{n+2, E}^{0}$. Then

$$
\left\langle F,-A_{0, E}^{2}(F)\right\rangle_{n, E}=\left\|A_{0, E}(F)\right\|_{n, E}^{2}=\lim _{\lambda \rightarrow 0^{+}}\left\|\frac{I-\left(C_{\lambda} T_{E}\right)^{q_{E}}}{\lambda}(F)\right\|_{n, E}^{2} .
$$

On the other hand Lemma 3.5 implies that the operator $I-\left(C_{\lambda} T_{E}\right)^{q_{E}}$ is invertible on $H_{n, E}^{0}$ for all $\lambda, 0<\lambda \leqq 1$ and

$$
\left\|\left[I-\left(C_{\lambda} T_{E}\right)^{q_{E}}\right]^{-1}(F)\right\|_{n, E} \leqq C \lambda\|F\|_{n, E}, \quad F \in H_{n, E}^{0},
$$

where $C$ is some constant independent of $\lambda$. Combining the last two relations together we get that

$$
\left\langle F,-A_{0, E}^{2}(F)\right\rangle_{n, E} \geqq C\|F\|_{n, E}^{2} .
$$

It remains to show that $\xi_{E}^{(0)}$ as defined through (3.11) is an eigenvector that corresponds to the eigenvalue 0 . However this is a matter of a simple straightforward computation and is left to the reader. 
We finish this section by presenting our proof for Theorem 1.2. Let $E$ be $\ell$-regular and let $\xi_{\lambda, E}$ be the unique solution of (2.9) such that $\xi_{\lambda, E}(0)=1$ and let $n$ be a fixed but arbitrary positive integer. We will show that the function

$$
\mathbb{R} \ni \lambda \mapsto \xi_{\lambda, E} \in H_{k, E}
$$

has an asymptotic expansion to $n^{\text {th }}$ order at $\lambda=0$ for all $k=0,1,2, \ldots$, and that its coefficients $\left\{\xi_{E}^{(0)}\right\}_{i=1, \ldots, n}$ satisfy the equations

$$
\begin{aligned}
& \xi_{E}^{(0)}(0)=1, \quad \xi_{E}^{(i)}(0)=0, \quad i=1,2, \ldots, n \\
& A_{0, E} \xi_{E}^{(0)}=0 \\
& (n+1) A_{0, E} \xi_{E}^{(n)}=-\sum_{k=2}^{n+1}\left(\begin{array}{c}
n+1 \\
k
\end{array}\right)\left(\frac{\partial^{k}}{\partial \lambda^{k}}\left(B_{\lambda}(0) T_{E}\right)^{q_{E}}\right)_{\lambda=0} \xi_{E}^{(n+1-k)}, n \geqq 1 .
\end{aligned}
$$

We will use induction in $n$.

$n=0$.

Let $F \in H_{k+4, E}$ and let $G \in H_{k, E}$. Using Taylor's theorem we get

$$
\begin{aligned}
\left\langle G,\left(B_{\lambda}(0) T_{E}\right)^{k_{E}}(F)\right\rangle_{k, E}= & \langle G, F\rangle_{k, E}+\lambda\left\langle G, A_{0, E}(F)\right\rangle_{k, E} \\
& +\lambda^{2}\left\langle G,\left(\frac{\partial^{2}}{\partial \lambda^{2}}\left(B_{\lambda}(0) T_{E}\right)^{q_{E}}\right)_{\lambda=c}(F)\right\rangle
\end{aligned}
$$

for some $c$ with $|c|<|\lambda|$. Replacing $F$ by $\xi_{\lambda, E}$ we get

$$
\left\langle G, A_{0, E}\left(\xi_{\lambda, E}-\xi_{E}^{(0)}\right)\right\rangle_{k, E}=-\lambda^{2}\left\langle G,\left[\frac{\partial^{2}}{\partial \lambda^{2}}\left(B_{\lambda}(0) T_{E}\right)^{k_{E}}\right]_{\lambda=c^{\prime}}\left(\xi_{k, E}\right)\right\rangle_{k, E}
$$

for some $c^{\prime}$ with $\left|c^{\prime}\right|<|\lambda|$. Since $\frac{\partial^{2}}{\partial \lambda^{2}}\left(B_{\lambda}(0) T_{E}\right)^{q_{E}}$ is a bounded operator from $H_{k+4, E}$ to $H_{k, E}$, we get from Lemma 3.1 that

$$
\left|\left\langle G, A_{0, E}\left(\xi_{\lambda, E}-\xi_{E}^{(0)}\right)\right\rangle_{k, E}\right| \leqq M|\lambda|\|G\|_{k, E}\left\|\xi_{\lambda, E}\right\|_{k, E}
$$

for all $0 \leqq \lambda \leqq 1$ and some $M>0$ independent at $\lambda$. Since $G$ was arbitrary we conclude from Theorem 3.6 that

$$
\left\|\xi_{\lambda, E}-\xi_{E}^{(0)}\right\|_{k, E} \leqq M|\lambda|\left\|\xi_{\lambda, E}\right\|_{k, E}
$$

for all $0 \leqq \lambda \leqq 1$ and for some $M>0$ independent of $\lambda$. Thus $\xi_{\lambda, E} \rightarrow \xi_{E}^{(0)}$ in $H_{k, E}$ for all $k=0,1,2, \ldots$ and the proof in the case $n=0$ is complete. $n \rightarrow n+1$.

Let us assume that the result is true for $n$ and let $G \in H_{k, E}$. Using Taylor's theorem as before we get

$$
\begin{aligned}
0= & \left\langle G, \lambda A_{0, E}\left(\xi_{\lambda, E}\right)\right\rangle_{\lambda, E}+\left\langle G, \sum_{i=2}^{n+2} \frac{\lambda^{i}}{i !}\left[\frac{\partial^{i}}{\partial \lambda^{i}}\left(B_{\lambda}(0) T_{E}\right)^{q_{E}}\right]_{\lambda=0}\left(\xi_{\lambda, E}\right)\right\rangle_{k, E} \\
& +\left\langle G, \frac{\lambda^{n+3}}{(n+3) !}\left(\frac{\partial^{n+3}}{\partial \lambda^{n+3}}\left(B_{\lambda}(0) T_{E}\right)^{q_{E}}\right)_{\lambda=0}\left(\xi_{\lambda, E}\right)\right\rangle_{k, E}
\end{aligned}
$$


for some $|c|<|\lambda|$. Substituting

$$
\xi_{\lambda, E}=\sum_{i=0}^{n} \xi_{E}^{(i)} \frac{\lambda^{i}}{i !}+o\left(\lambda^{n}\right)
$$

into the equation above and rearranging the summations we get that for small $\lambda$

$$
\begin{gathered}
\left.\left\langle G, \lambda A_{0, E}\left(\xi_{\lambda, E}\right)\right\rangle+\sum_{i=2}^{n+1} \frac{\lambda^{i}}{i !}\left[\sum_{j=2}^{i}\left(\begin{array}{c}
i \\
j
\end{array}\right)\left(\frac{\partial^{j}}{\partial \lambda^{j}}\left(B_{\lambda}(0) T_{E}\right)^{q_{E}}\right)_{\lambda=0}\left(\xi_{E}^{(i-j)}\right)\right]+O_{n, E}(\lambda)\right\rangle_{k, E} \\
=\left\langle G, \frac{\lambda^{n+2}}{(n+2) !} \sum_{j=2}^{n+2}\left(\begin{array}{c}
n+2 \\
j
\end{array}\right)\left(\frac{\partial^{j}}{\partial \lambda^{j}}\left(B_{\lambda}(0) T_{E}\right)^{q_{E}}\right)_{\lambda=0}\left(\xi_{E}^{(n+2-j)}\right)\right\rangle_{k, E},
\end{gathered}
$$

where $O_{n, E}(\lambda)$ contains all the other terms which are of order higher than $\lambda^{n+2}$. Using Eq. (3.12) we can now rewrite the equations above as

$$
\begin{gathered}
\left\langle G,(n+2) ! A_{0, E}\left[\frac{1}{\lambda^{n+1}}\left(\xi_{\lambda, E}-\sum_{i=0}^{n} \frac{\lambda^{i}}{i !} \xi_{E}^{(i)}\right)\right]+\frac{(n+2) !}{\lambda^{n+2}} O_{n, E}(\lambda)\right\rangle_{k, E} \\
=\left\langle G, \sum_{j=2}^{n+2}\left(\begin{array}{c}
n+2 \\
j
\end{array}\right)\left(\frac{\partial^{j}}{\partial \lambda^{j}}\left(B_{\lambda}(0) T_{E}\right)^{q_{E}}\right)_{\lambda=0}\left(\xi_{E}^{(n+2-j)}\right)\right\rangle_{k, E}
\end{gathered}
$$

for some $c$ such that $|c|<|\lambda|$.

Let $\widetilde{\xi}_{E}^{(n+1)}$ be the unique solution of

$$
(n+2) A_{0, E} \xi^{(n+1)}=-\sum_{j=2}^{n+2}\left(\begin{array}{c}
n+2 \\
j
\end{array}\right)\left(\frac{\partial^{j}}{\partial \lambda^{j}}\left(B_{\lambda}(0) T_{E}\right)^{q_{E}}\right)_{\lambda=0}\left(\xi_{E}^{(n+2-j)}\right) .
$$

We can now rewrite Eq. (3.14) as

$$
\begin{aligned}
& \left\langle G, A_{0, E}\left[\frac{1}{\lambda^{n+1}}\left(\xi_{\lambda, E}-\sum_{i=0}^{n} \frac{\lambda^{i}}{i !} \xi_{E}^{(i)}\right)-\frac{1}{(n+1) !} \tilde{\xi}_{E}^{(n+1)}\right]\right\rangle_{k, E} \\
& =-\left\langle G, \frac{1}{\lambda^{n+2}} O_{n, \mathrm{E}}(\lambda)\right\rangle_{k, E} .
\end{aligned}
$$

The key estimate of Lemma 3.1 however allows us to estimate $O_{n, \mathrm{E}}(\lambda)$. Indeed one can easily check in (3.13) that even though $O_{n, \mathrm{E}}(\lambda)$ may apriori depend on $G$ has to satisfy the bound

$$
\left\|O_{n, \mathrm{E}}(\lambda)\right\|_{k, E} \leqq M|\lambda|^{n+3}\left\|\xi_{\lambda, E}\right\|_{k, E}+|\lambda|^{n+2} \varepsilon_{\lambda}
$$

for all $\lambda, 0<|\lambda| \leqq 1$, some $M>0$ independent of $\lambda$ and where $\varepsilon_{\lambda}$ converge to zero as $\lambda \rightarrow 0$.

Using this crucial bound above we get from Theorem 3.6 that

$$
\left\|\frac{1}{\lambda^{n+1}}\left(\xi_{\lambda, E}-\sum_{i=0}^{n} \frac{\lambda^{i}}{i !} \xi_{E}^{(i)}\right)-\frac{1}{(n+1) !} \tilde{\xi}_{E}^{(n+1)}\right\|_{k, E} \leqq M|\lambda|\left\|\xi_{\lambda, E}\right\|_{k, E}+\varepsilon_{\lambda}
$$

for all $\lambda, 0<|\lambda| \leqq 1$, and where $M$ and $\varepsilon_{\lambda}$ are as before. 
Multiplying by $|\lambda|^{n+1}$ both sides of the inequality above and using the triangle inequality we obtain that

$$
\begin{gathered}
\left(1-M|\lambda|^{n+2}\right)\left\|\frac{1}{\lambda^{n+1}}\left(\xi_{\lambda, E}-\sum_{i=0}^{n} \frac{\lambda^{i}}{i !} \xi_{E}^{(i)}\right)-\frac{1}{(n+1) !} \widetilde{\xi}_{E}^{(n+1)}\right\|_{k, E} \\
\leqq M|\lambda|\left\|\sum_{i=0}^{n} \frac{\lambda^{i}}{i !} \xi_{E}^{(i)}+\frac{\lambda^{n+1}}{(n+1) !} \widetilde{\xi}_{E}^{(n+1)}\right\|_{k, E}+\varepsilon_{\lambda}
\end{gathered}
$$

for all $\lambda, 0<|\lambda| \leqq 1$, and where $M, \varepsilon_{\lambda}$ are as before. Thus

$$
\frac{1}{\lambda^{n+1}}\left(\xi_{\lambda, E}-\sum_{i=0}^{n} \frac{\lambda^{i}}{i !} \xi_{E}^{(i)}\right) \rightarrow \frac{1}{(n+1) !} \tilde{\xi}_{E}^{(n+1)}
$$

in $H_{k, E}$ for all $k=0,1, \ldots$ as $\lambda \rightarrow 0$ and $\tilde{\xi}_{E}^{(n+1)}=\xi_{E}^{n+1}$.

\section{Interpolation Bounds}

In this last section we derive the key technical estimate described in Lemma 3.1. Since for $\lambda$ away from zero the result follows through standard perturbation techniques developed in [11] we restrict ourselves to the case where $\lambda$ is very small. Without loss of generality we can also assume, via a standard approximation argument, that the first and second moments of $\mu$ are finite. Our proof is mainly based on the Calderon-Lions abstract interpolation theory as described by Theorem IX.20 of [12].

We begin by introducing a family of positive norms \|\|$_{n, b, c}^{E}, n=1,2, \ldots$, $b, c \in \mathbb{R},|E|<1+\cos \frac{\pi \ell}{\ell+1}$ on $\mathscr{S}\left(\mathbb{R}^{2 \ell}\right)$ through the following equations:

$$
\begin{aligned}
\left(\|f\|_{n, b, c}^{E}\right)^{2}= & \sum_{k_{1}, \ldots, k_{n}=1}^{2 \ell} \sum_{i_{1}, \ldots, i_{n}=1}^{2} \\
& \times\left\|\prod_{m=1}^{n-1}\left(1+b^{2} \varphi_{i}^{4}\right)^{c}\left[\prod_{m=1}^{n-1}\left[A_{E}^{1 / 2}\left(Q_{i_{m}}\right)\right]_{k_{m}^{(F)}}\right]\right\|_{L^{2}\left(\mathbb{R}^{2 \ell}, d^{2 \ell} \varphi\right)}^{2},
\end{aligned}
$$

where we have used the covention $\varphi_{i}^{4}=\left(\varphi_{i}^{2}\right)=\left(\varphi_{1} \cdot \varphi_{i}\right)^{2}, i=1,2, \ldots, \ell$.

Definition 4.1. Let $n, \ell$ be positive integers, let $c, b$ be positive real numbers and let $|E|<1+\cos \frac{\pi \ell}{\ell+1}$. We define the weighted supersymmetric Hilbert space $H_{n, E}^{c, b}$ to be the completion of $\mathscr{S}\left(\mathscr{L}_{\ell}\right)$ under the norm \|\|$_{n, E}^{c, b}$, where

$$
\left(\|F\|_{n, E}^{c, b}\right)^{2}=\sum_{a \in \mathcal{O}_{\ell}} \sum_{m=1}^{n}\left(\left\|\left(A_{E}^{1 / 2} F\right)_{a}\right\|_{m, b, c}^{E}\right)^{2}+|F(0)|^{2}, F \in \mathscr{P}\left(\mathscr{L}_{\ell}\right)
$$

Let $n$ be positive integer and let $c, b$ be positive real numbers with $|b|<1$. As in Sect. II it is easy to see that if $I$ is a compact set of $\left(-1-\cos \frac{\pi \ell}{\ell+1}\right.$, $\left.1+\cos \frac{\pi \ell}{\ell+1}\right)$

$$
C_{I}^{\prime}\|F\|_{n, 0}^{c, b} \leqq\|F\|_{n, E}^{c, b} \leqq C_{I}\|F\|_{n, 0}^{c, b}
$$


for all $F \in \mathscr{S}\left(\mathscr{L}_{\ell}\right)$ and for some $C_{I}, C_{I}^{\prime}$ positive constants that depend only on the set $I$.

Let $\sigma$ be a real positive number we will make use of the operator $M_{\sigma}$ which is defined by

$$
\left[M_{\sigma}(F)\right]_{a}\left(\varphi_{1}, \ldots, \varphi_{n}\right)=\prod_{i=1}^{n}\left(1+\varphi_{i}^{4}\right)^{\sigma} F_{a}\left(\varphi_{1}, \ldots, \varphi_{n}\right), F \in C^{1}\left(\mathscr{L}_{\ell}\right)
$$

Lemma 4.2. Let $h$ and $\alpha$ be as in the hypothesis of Theorem 1.2. Let $j$ be a positive integer, $|E|<1+\cos \frac{\pi \ell}{\ell+1}$ and let $F \in H_{j, E}$. If $k_{1}, \ldots, k_{j}$ and $i_{1}, \ldots, i_{j}$ are two sequences with values in $\{1,2, \ldots, 2 \ell\}$ and $\{1,2\}$ respectively, then for all $\lambda,|\lambda|<1$, $y \in \mathbb{R}$ and $\alpha^{\prime} \leqq \alpha$,

$$
\begin{aligned}
e^{-y^{2}} & \left(\prod_{m=1}^{j-1}\left[A_{E}^{1 / 2} Q_{i_{m}}\right]_{k_{m}} M_{\lambda^{2}\left(-i y+\alpha^{\prime} / 4\right)} A_{E}^{1 / 2} B_{\lambda}(0)\right)(F) \\
= & e^{-y^{2}}\left(M_{\lambda^{2}\left(-i y+\alpha^{\prime} / 4\right)} A_{\mathrm{E}}^{1 / 2}\right) \mathbb{E}_{U}\left\{b_{\lambda U}\left(\prod_{m=1}^{j-1}\left[A_{E}^{1 / 2} Q_{i_{m}}\right]_{k_{m}} A_{E}^{1 / 2}\right)(F)\right\} \\
& +\lambda M_{\lambda^{2}\left(-i y+\alpha^{\prime} / 4\right)} A_{\mathrm{E}}^{1 / 2}\left(\mathbb{E}_{U}\left\{b_{\lambda U}\left(U_{1} O_{1}(F)+\ldots+U_{\ell} O_{\ell}(F)\right\}\right)+\lambda^{2} O_{0}(F),\right.
\end{aligned}
$$

where $O_{0}(F), \ldots, O_{\ell}(F)$ are super functions that satisfy the inequalities

$$
\left\|Q_{i}(F)\right\| \underset{1, E}{c, b} \leqq M\|F\| \underset{j, E}{c, b}
$$

for all $i=1, \ldots, \ell$ and $c, b$ positive real numbers some $M$ independent of $c, b$ and $F$ and where $U_{1}, \ldots, U_{\ell}$ are the diagonal matrix elements of $U$.

Proof. The proof follows immediately from an explicit straightforward computation and is left to the reader.

Lemma 4.3. Let $E, h$ and $\alpha$ be as in the previous lemma and let $n$ be a positive integer. let us assume (for the moment) that the mean of the distribution $\mu$ of the single sited potential is zero. Then

$$
e^{-y^{2}}\left\|\left(M_{\lambda^{2}(-i y+\alpha / 4)} B_{\lambda}(0)\right)(F)\right\|_{n, E} \leqq\|F\|_{n, E}+M \lambda^{2}\|F\|_{n, E}^{1, \lambda}
$$

for all $\lambda,|\lambda|<1, y \in \mathbb{R}$, some $M>0$ independent of $\lambda$ and all $F \in H_{n, E}$ and

$$
\left\|\left(B_{\lambda}(0)\right)(F)\right\|_{n, E}^{c, b} \leqq\left(1+M \lambda^{2}\right)\|F\|_{n, E}^{c, b}
$$

for all $\lambda,|\lambda|<1, b, c$ positive real numbers, some $M>0$ independent of $c, b$ and all $F \in H_{n, E}$.

Proof. Let $c, b$ be two positive real numbers. Let $j$ be an integer from $\{1,2, \ldots, n\}$ and let $k_{1}, \ldots, k_{j-1}$ and $i_{1}, \ldots, j_{j-1}$ be two sequences with values in $\left\{1,2, \ldots, 2 \ell\right.$ ) and $\{1,2\}$ respectively. Using Eq. (4.1) with $\alpha^{\prime}=y=0$ we get that if $F \in H_{j, E}$, then 


$$
\begin{aligned}
e^{-y^{2}} & \prod_{i=1}^{\ell}\left(1+b^{2} \varphi_{i}^{4}\right)^{c}\left[\prod_{m=1}^{j-1}\left[A_{E}^{1 / 2} Q_{i_{m}}\right]_{k_{m}} A_{E}^{1 / 2} B_{\lambda}(0)\right](F) \\
= & e^{-y^{2}} \mathbb{E}_{U}\left\{\prod_{i=1}^{\ell}\left(1+b^{2} \varphi_{i}^{4}\right)^{c} b_{\lambda U}\left[\prod_{m=1}^{j-1}\left[A_{E}^{1 / 2} Q_{i_{m}}\right]_{k_{m}} A_{\mathrm{E}}^{1 / 2}\right](F)\right. \\
& +\prod_{i=1}^{\ell}\left(1+b^{2} \varphi_{i}^{4}\right)^{c}\left[\lambda b_{\lambda U}\left(U_{1} O_{1}(F)+\ldots+U_{\ell} O_{\ell}(F)\right]\right\} \\
& +\lambda^{2} \prod_{i=1}^{\ell}\left(1+b^{2} \varphi_{i}^{4}\right)^{c} O_{0}(F)
\end{aligned}
$$

for all $\lambda,|\lambda|<1$. One can easily check now that by taking $L^{2}\left(\mathbb{R}^{2 \ell}, d^{2 \ell} \varphi\right)$ norm both sides of the equation above and using Jensen inequality (4.3) follows easily from Definition 4.1.

Using once again Eq. (4.1) with $\alpha^{\prime}=\alpha$ and $y \in \mathbb{R}$ we get

$$
\begin{aligned}
e^{-y^{2}}\left(\prod_{m=1}^{j-1}\left[A_{E}^{1 / 2} Q_{i_{m}}\right]_{k_{m}} M_{\lambda^{2}(-i y+\alpha / 4)} A_{E}^{1 / 2} B_{\lambda}(0)\right)(F) \\
=e^{-y^{2}} \mathbb{E}_{\tilde{U}}\left\{b_{\lambda \tilde{U}}\left(\prod_{i=1}^{\ell}\left(1+\lambda^{2} \varphi_{i}^{4}\right)^{\alpha / 4} h\left(\lambda \varphi_{i}^{2}\right)\right)\right. \\
\left.\times\left[M_{\lambda^{2}(-i y+\alpha / 4)}\left(\prod_{m=1}^{j-1}\left[A_{E}^{1 / 2} Q_{i_{m}}\right]_{k_{m}}\right) A_{E}^{1 / 2}\right](F)\right\} \\
+\lambda \mathbb{E}_{\tilde{U}}\left\{b_{\lambda \tilde{U}} \sum_{i=1}^{\ell} \tilde{U}_{i}\left[h^{\prime}\left(\lambda \varphi_{i}^{2}\right) \frac{\left(1+\lambda^{2} \varphi_{i}^{4}\right)}{\lambda \varphi_{i}^{2}} \prod_{m=1}^{j-1}\left(1+\lambda^{2} \varphi_{i}^{4}\right)^{\alpha / 4}\right]\right. \\
\left.\times M_{\lambda^{2}(-i y+\alpha / 4)}\left(O_{i}(F)\right)\right\}+\lambda^{2} O_{0}(F),
\end{aligned}
$$

where $\tilde{U}$ is equal to -1 times the $\ell \times \ell$ diagonal random matrix whose diagonal elements $\widetilde{U}_{1}, \ldots, \widetilde{U}_{\ell}$ are independent copies of the two sited gamma distribution with parameters $1, \alpha / 4$. The proof of (4.2) now follows as, in the previous case, from Jensen inequality and Definition 4.1.

Let $n$ be a positive integer and let $|E|<1+\cos \frac{\pi \ell}{\ell+1}$. We introduce a sequence of interpolating spaces $L_{n, E}^{k}(t), 0 \leqq t \leqq 1, k=0,1,2, \ldots$ inductively by defining $L_{n, E}^{k}(t)$ to be the $t^{\text {th }}$ interpolation space between $L_{n, E}^{k-1}(t)$ and $H_{n+2, E}$, where $L_{n, E}^{1}(0)=H_{n, E}$ and $L_{n, E}^{1}(1)=H_{n+2, E}$.

Lemma 4.4. Let $n, E$ be as above, then $L_{n, E}^{k}(t)$ can be continuously imbedded in $H_{n, E}^{1 / 2\left[1-(1-t)^{k}\right], 1}$ for all $k=0,1,2, \ldots$ and

$$
\|F\|_{n, E}^{1 / 2\left[1-(1-t)^{k}\right], 1} \leqq M\|F\|_{L_{n, E}^{k}(t)}
$$

for all $t, 0 \leqq t \leqq 1, F \in L_{n, E}^{k}(t)$ and some $M>0$ independent of $t$ and $k$.

Proof. Let $j$ be an integer from $\{1, \ldots, n\}$, and let $k_{1}, \ldots, k_{j-1}$ and $i_{1}, \ldots, i_{j-1}$ be two finite sequences with values in $\{1,2, \ldots, 2 \ell\}$ and $\{0,1\}$ respectively. Clearly

$$
\left\|\left(\prod_{m=1}^{j-1}\left[A_{E}^{1 / 2} Q_{i_{m}}\right]_{k_{m}}\right)(F)\right\|_{1, E}^{0,1} \leqq\|F\|_{n, E}
$$


and

$$
\left\|\left(\prod_{m=1}^{j-1}\left[A_{E}^{1 / 2} Q_{i_{m}}\right]_{k_{m}}\right)(F)\right\|_{1, E}^{1 / 2,1} \leqq M\|F\|_{n+2, E}
$$

for all $F \in \mathscr{S}\left(\mathscr{L}_{\ell}\right)$ and some $M>0$ independent of $j$. Interpolating between the inequalities above and taking into account that the $t^{\text {th }}$ interpolation space between $H_{1, E}^{1 / 2,1}$ and $H_{1, E}^{0,1}$ is $H_{1, E}^{t / 2,1}$ we obtain that

$$
\|F\|_{n, E}^{t / 2,1} \leqq M\|F\|_{L_{n, E}^{1}(t)}
$$

for all $F \in \mathscr{S}\left(\mathscr{L}_{t}\right), t, 0 \leqq t \leqq 1$ and some $M>0$ independent of $t$.

The result now follows by iterating the procedure above and from the fact that the $t^{\text {th }}$ interpolation space of $H_{1, E}^{1 / 2\left[1-(1-t)^{k}\right]}$ and $H_{1, E}^{1 / 2,1}$ is $H_{1, E}^{1 / 2\left[1-(1-t)^{k+1}\right]}$.

For our next lemma we will need a rather smaller weighted supersymmetric Hilbert space $H_{n, E, m}$ which we now introduce as the completion of $\mathscr{S}\left(\mathscr{L}_{\ell}\right)$ under the norm $\|\cdot\|_{n, E, m}$, where

$$
\left(\|F\|_{n, E, m}\right)^{2}=\sum_{\alpha \in \mathscr{O}_{\ell}} \sum_{j=1}^{n+m}\left(\left\|\left(A_{E}^{1 / 2} F\right)_{a}\right\|_{j}^{E}\right)^{2}|\lambda|^{x_{0}(j-n)}+|F(0)|^{2}
$$
$n, m$ are positive integers, $|E|<1+\cos \frac{\pi \ell}{\ell+1}$ and $\mathscr{X}_{0}$ denotes the characteristic
function of $(0,+\infty)$.

We shall also make use of a sequence of the interpolating spaces $L_{n, E, m}^{k}(t)$, $0 \leqq t \leqq 1, k=0,1,2, \ldots$ which are inductively defined by setting $L_{n, E, m}^{k}(t)$ to be the $t^{\text {th }}$ interpolation space between $L_{n, E, m}^{k-1}(t)$ and $H_{n, E, 8 m}$, where $L_{n, E, m}^{1}(0)=H_{n, E, 0}$ and $L_{n, E, m}^{1}(1)=H_{n, E, 8 m}$.

Lemma 4.5. Let $\alpha$ and $h$ be as in the hypothesis of Theorem 1.2. For every $m, n$ positive integers there exists $k_{0}$ positive integer such that

$$
\begin{aligned}
& \left\|\left(T_{E} B_{\lambda}(0)\right)^{2 k_{0}}(F)\right\|_{n, E}^{m, \lambda} \\
& \quad+\left\|e^{z^{2}}\left(T_{E} M_{\lambda^{2}(-z+\alpha / 4)} B_{\lambda}(0)\left(T_{E} B_{\lambda}(0)\right)^{2 k_{0}+1}\right)(F)\right\|_{n, E}^{m, \lambda} \leqq M\|F\|_{n, E}
\end{aligned}
$$

for all $\lambda,|\lambda|<1$. $F \in H_{n, E}$ energies $E$ such that $|E|<1+\cos \frac{\pi \ell}{\ell+1}, z \in\{x+i y$, $x=0,1, y \in \mathbb{R}\}$ and for some $M$ positive constant independent of $\lambda$.

Proof. Let $|E|<1+\cos \frac{\pi \ell}{\ell+1}$ and let $m, n$ be two positive integers. Consider the operator valued function $G_{\lambda}$ which is defined on the strip $S=\{x+i y, 0 \leqq x \leqq 1$, $y \in \mathbb{R}\}$ through the formula

$$
G_{\lambda}(z)=e^{z^{2}}\left(T_{E} B_{\lambda}(0) M_{-8 m z+\alpha / 2}^{\lambda}\right)^{2},
$$

where $M_{c}^{\lambda}$ is the multiplication operator defined by

$$
\left[M_{. c}^{\lambda}(F)\right]_{a}\left(\varphi_{1}, \ldots, \varphi_{n}\right)=\prod_{i=1}\left(1+\lambda^{2} \varphi_{i}^{4}\right)^{c / 4} F_{a}\left(\varphi_{1}, \ldots, \varphi_{n}\right), \quad F \in C^{1}\left(\mathscr{L}_{\ell}\right) .
$$

Clearly $G_{\lambda}(z)$ is a bounded operator on $H_{n, E, 8 m}$ for all $z \in S$ and $G_{\lambda}$ is norm continuous on $S$ while being analytic in the interior of $S$. Moreover through a 
straightforward computation similar to the one used in part (iii) of Proposition IV.1.4 of [11] one can easily show that

$$
\begin{gathered}
\left\|\left(G_{\lambda}(i y)\right)(F)\right\|_{n, E, 0}=\|\left(G_{\lambda}(i y)(F)\left\|_{n, E} \leqq M\right\| F \|_{n, E},\right. \\
\left\|\left(G_{\lambda}(1+i y)\right)(F)\right\|_{n, E, 8 m} \leqq M\|F\|_{n, E},
\end{gathered}
$$

and

$$
\|\left(G_{\lambda}\left(\frac{\alpha}{16 m}\right)(F)\left\|_{n, E, 8 m}=e^{\left(\frac{\alpha}{16 m}\right)^{2}}\right\|\left(T_{E} B_{\lambda}(0)\right)^{2}(F)\left\|_{n, E, 8 m} \leqq M\right\| F \|_{n, E, 8 m}\right.
$$

for all $y \in \mathbb{R}, \lambda,|\lambda|<1, F \in H_{n, E, 8 m}$ and some $M>0$ independent of $y$ and $\lambda$.

Interpolating between the first and second inequalities we get that

$$
\left\|\left(T_{E} B_{\lambda}(0)\right)^{2}(F)\right\| L_{n, E, m}^{1}\left(\frac{\alpha}{16 m}\right) \leqq M\|F\|_{n, E} .
$$

Interpolating between the last two inequalities we get that

$$
\left\|\left(T_{E} B_{\lambda}(0)\right)^{2}(F)\right\| L_{n, E, m}^{2}\left(\frac{a}{16 m}\right) \leqq M\|F\| L_{n, E, m}^{1}\left(\frac{\alpha}{16 m}\right)
$$

for all $\lambda,|\lambda|<1, F \in L_{n, E, m}^{1}\left(\frac{\alpha}{16 m}\right)$ and some $M>0$ independent of $\lambda$. Iterating this procedure $k$ times we obtain

$$
\left\|\left(T_{E} B_{\lambda}(0)\right)^{2 k}(F)\right\| L_{n, E, m}^{k}\left(\frac{\alpha}{16 m}\right) \leqq M^{k}\|F\|_{n, E}
$$

for all $\lambda,|\lambda|<1, F \in H_{n, E}$ and some $M>0$ independent of $\lambda$. Using essentially the same arguments with the ones presented in the proof of Lemma 4.4, one can show that $H_{n, E}^{2 m\left[1-(1-t)^{k}\right], \lambda}$ is canonically imbedded in $L_{n, E, m}^{k}(t)$. Thus we conclude that if we set $k_{0}=\left[\frac{16 m \ln 2}{\alpha}\right]+1$, then

$$
\left\|\left(T_{E} B_{\lambda}(0)\right)^{2 k_{0}}(F)\right\|_{n, E}^{m, \lambda} \leqq M^{k_{0}}\|F\|_{n, E}
$$

for all $\lambda,|\lambda|<1, F \in H_{n, E}$ and some $M>0$ independent of $\lambda$. Trivially the same series of arguments could be applied for $e^{z^{2}} T_{E} M_{\lambda^{2}(-i z+\alpha / 4)} B_{\lambda}(0) T_{E} B_{\lambda}(0)$ and the result follows.

Lemma 4.6. Let $n, E, k_{0}, \alpha$ and $\mu$ be as in the previous lemmas. Consider the operator valued function $\Gamma_{\lambda}$ which is defined on the strip $S_{\lambda}=\left\{x+i y: 0 \leqq x \leqq 1 / \lambda^{2}\right.$, $y \in \mathbb{R}\}$ through the formula

$$
\Gamma_{\lambda}(z)=e^{z^{2}}\left(T_{E} B_{\lambda}(0) M_{\lambda^{2}(-z+\alpha / 4)}\right)^{2}\left(T_{E} B_{\lambda}(0)\right)^{2 k_{0}+1},
$$

where $k_{0}$ is the integer specified in Lemma 4.5 for the case $m=1$.

The following statements are true for all $\lambda, 0<|\lambda|<1$ :

(i) $\Gamma_{\lambda}(z)$ is a bounded operator on $H_{n+2, E}$ for all $z \in S_{\lambda}$.

(ii) $\Gamma_{\lambda}$ is analytic in the interior of $S_{\lambda}$ and norm continuous on $S_{\lambda}$.

(iii) Let $F \in H_{n, E}$ then

$$
\left\|\left(\Gamma_{\lambda}\left(i y+1 / \lambda^{2}\right)\right)(F)\right\|_{n+2, E} \leqq M\|F\|_{n, E},
$$

and

$$
\left\|\left(\Gamma_{\lambda}(i y)\right)(F)\right\|_{n+2, E} \leqq e^{c \lambda^{2}}\|F\|_{n+2, E}
$$

for all $y \in \mathbb{R}$ and $M, c$ positive constants independent of $y$ and $\lambda$.

Proof. (i) and (ii) are straightforward. Through an easy computation similar to the one used in part (iii) of Proposition IV.1.4 of [11] one can easily show that 


$$
\begin{aligned}
& e^{\left(i y+1 / \lambda^{2}\right)^{2}}\left\|\left(M_{\lambda^{2}\left[\alpha / 4-\left(i y+1 / \lambda^{2}\right)\right]} B_{\lambda}(0) T_{E} M_{\lambda^{2}\left[\alpha / 4-\left(i y+1 / \lambda^{2}\right)\right]} B_{\lambda}(0)\right)(F)\right\|_{n+2, E} \\
& \leqq M\|F\|_{n, E}
\end{aligned}
$$

for all $F \in H_{n, E}, \lambda, 0<|\lambda|<1, y \in \mathbb{R}$ and for some $M>0$ independent of $\lambda$ and $y$. Thus the first inequality of part (iii) follows from the fact that $T_{E}$ and $B_{\lambda}(0)$ are bounded operators. $H_{n+2, E}$,

Let $F \in H_{n, E}$ from Lemma 4.3 we conclude that since $T_{E}$ is an isometry in

$$
\begin{aligned}
\left\|\left(\Gamma_{\lambda}(i y)\right)(F)\right\|_{n+2, E} \leqq & \|\left(B_{\lambda}(0) M_{\lambda^{2}(-i y+\alpha / 4)}\left(T_{E} B_{\lambda}(0)\right)^{\left.2 k_{0}+1\right)}(F) \|_{n+2, E}\right. \\
& +M \lambda^{2}\|F\|_{n+2, E}
\end{aligned}
$$

for all $\lambda, 0<|\lambda|<1$ and some $M>0$ independent of $y$ and $\lambda$. Thus

$$
\begin{aligned}
\left\|\left(\Gamma_{\lambda}(i y)\right)(F)\right\|_{n+2, E} \leqq & \left\|\left(T_{E} B_{\lambda}(0)\right)^{2 k_{0}+1}(F)\right\|_{n+2, E}+M \lambda^{2}\|F\|_{n+2, E} \\
& +M \lambda^{2}\left\|\left(T_{E} B_{\lambda}(0)\right)^{2 k_{0}}\left(T_{E} B_{\lambda}(0)\right)(F)\right\|_{n+2, E}^{1, \lambda}
\end{aligned}
$$

for all $\lambda, 0<|\lambda|<1$ and some $M>0$ independent of $y$ and $\lambda$. The rest of the proof now follows from inequality (4.3) and the fact that $T_{E}$ is an isometry.

We finish this section by combing Lemma 4.4 and Lemma 4.6 into a proof of Lemma 3.1. Let $n$ be a position integer and let $|E|<1+\cos \frac{\pi \ell}{\ell+1}$. Theorem IX.20 of [12] and Lemma 4.6 imply that if the mean of the distribution $\mu$ is zero then

$$
\left\|\left(T_{E} B_{\lambda}(0)\right)^{2 k_{0}+3}(F)\right\|_{L^{1}{ }_{n, E}\left(\alpha \lambda^{2} / 4\right)} \leqq e^{c_{1} \lambda^{2}}\|F\|_{n, E}
$$

for all $F \in L_{n, E}^{1}\left(\alpha \lambda^{2} / 4\right), \lambda$ with $0<|\lambda|<1$ and for some positive constant $c_{1}$. On the other hand, since $T_{E}$ is an isometry on $H_{n+2, E}$ we conclude from Lemma 4.3 that

$$
\left\|\left(T_{E} B_{\lambda}(0)\right)^{2 k_{0}+3}(F)\right\|_{n+2, E} \leqq e^{c_{2} \lambda^{2}}\|F\|_{n+2, E}
$$

for all $F \in H_{n+2, E}, \lambda$, with $0<|\lambda|<1$ and for some positive constant $c_{2}$. Interpolation between relations (4.4) and (4.5) yields

$$
\left\|\left(T_{E} B_{\lambda}(0)\right)^{2 k_{0}+3}(F)\right\|_{L_{n, E}^{2}\left(\alpha \lambda^{2} / 4\right)} \leqq e^{c \lambda^{2}}\|F\|_{L_{n, E}^{1}\left(\alpha \lambda^{2} / 4\right)}
$$

for all $F \in L_{n, E}^{1}\left(\alpha \lambda^{2} / 4\right), \lambda$ with $0<|\lambda|<1$ and where $c=\max \left(c_{1}, c_{2}\right)$.

Iterating this interpolation in the same spirit of the proof of Lemma 4.5 we obtain

$$
\left\|\left(T_{E} B_{\lambda}(0)\right)^{k\left(2 k_{0}+3\right)}(F)\right\|_{L_{n, E}^{k}\left(\alpha \lambda^{2} / 4\right)} \leqq e^{c k \lambda^{2}}\|F\|_{n, E}
$$

for all $F \in H_{n, E}$ and $k=0,1,2, \ldots$.

If $k>\log 16 / \alpha \lambda^{2}$ however, we conclude from Lemma 4.4 that

$$
\left\|\left(T_{E} B_{\lambda}(0)\right)^{k\left(2 k_{0}+3\right)}(F)\right\|_{n, E}^{1 / 4,1} \leqq M e^{c k \lambda^{2}}\|F\|_{n, E}
$$

for all $F \in H_{n, E}, \lambda$, with $0<|\lambda|<1$. Thus we have shown that if $\mu$ is centered and under the hypothesis of Theorem 1.2, there exists an $M>0$ such that for every $\lambda$ with $0<|\lambda|<1$ there exists an integer $P_{\lambda}$ such that

for all $F \in H_{n, E}$.

$$
\left\|\left(T_{E} B_{\lambda}(0)\right)^{P_{\lambda}}(F)\right\|_{n, E}^{1 / 4,1} \leqq M\|F\|_{n, E}
$$


Now let once again $k_{1}, \ldots, k_{n}$ and $i_{1}, \ldots, i_{n}$ be two finite sequences with values in $\{1,2, \ldots, 2 \ell\}$ and $\{0,1\}$ respectively. We will show that there exists an $M$ independent of these two sequences such that for all $\lambda, 0<|\lambda|<1$,

$$
\left\|\prod_{m=1}^{n}\left[A_{E}^{1 / 2} Q_{i_{m}}\right]_{k_{m}}\left[\left(T_{E} B_{\lambda}(0)\right)^{P_{\lambda}+1}(F)\right]\right\|_{1, E} \leqq\|F\|_{n, E}
$$

for all $F \in H_{n, E}$, and where $P_{\lambda}$ is the same exponent that appears in (4.8). In view of inequality (4.8) we need to study

$$
\left[Q_{i_{1}}\right]_{k_{1}}\left[\prod_{m=2}^{n}\left[A_{E}^{1 / 2} Q_{i_{m}}\right]_{k_{m}}\left[\left(T_{E} B_{\lambda}(0)\right)^{P_{\lambda}+1}(F)\right]\right]
$$

in the case $k_{1} \leqq \ell$. Indeed in this case the super function above is equal to

$$
(-i)^{n-1}\left[\left[Q_{i_{1}}\right]_{k_{1}} \mathscr{C}_{E} b_{\mathscr{E}_{E}} \mathscr{F}\left(\prod_{m=2}^{n}\left[\left(A_{E}^{1 / 2}\right) \mathscr{C}_{E}\left(Q_{i_{\mathrm{m}}}\right)\right]_{k_{m}}\right) B_{\lambda}(0)\left(T_{E} B_{\lambda}(0)\right)^{P_{\lambda}}\right](F)
$$

An elementary computation however shows that the super function above is equal to

$$
\begin{aligned}
& \left(\left[-2 \sum_{j=\ell}^{2 \ell}\left(\mathscr{E}_{E}\right)_{k_{1, j}}\left[Q_{i}\right]_{j}\right] \prod_{m=2}^{n}\left[A_{E}^{1 / 2} Q_{i_{m}}\right]_{k_{m}}\left[\left(T_{E} B_{\lambda}(0)\right)^{P_{\lambda}+1}\right]\right)(F) \\
& \quad-\left[\mathscr{C}_{E} b_{\mathscr{E}_{E}} \mathscr{F}\left[Q_{i_{1}}\right]_{k_{1}+\ell}\left(\prod_{m=2}^{n}\left[A_{E}^{1 / 2} \mathscr{C}_{E} Q_{i_{m}}\right]_{k_{m}}\right) B_{\lambda}(0)\left(T_{E} B_{\lambda}(0)\right)^{P_{\lambda}}\right](F) .
\end{aligned}
$$

Combining the latter with inequality (4.3) and (4.8) we can conclude that since $T_{E}$ is an isometry (4.9) holds. Thus we have shown that there exists an $M>0$ such that for all $\lambda, 0<|\lambda|<1$ there exists $P_{\lambda}$ positive integer such that

$$
\left\|\left(T_{E} B_{\lambda}(0)\right)^{P_{\lambda}+1}(F)\right\|_{n+1, E} \leqq M\|F\|_{n, E} .
$$

Since $T_{E}$ and $B_{\lambda}(0)$ are bounded operators on $H_{n+2}$ and $H_{n}$ we can rewrite (4.10) as

$$
\left\|\left(B_{\lambda}(0) T_{E}\right)^{P_{\lambda}+2}(F)\right\|_{n+1, E} \leqq M\|F\|_{n, E}
$$

for all $F \in H_{n, E}$, some $M$ independent of $\lambda$ and where $P_{\lambda}$ is the same exponent that appears in (4.10).

Notice that up to now the assumption that $\mu$ is centered was crucial. However if $\mu_{0}$ is the mean of $\mu$ one can easily see that

$$
\left\|\left(B_{\lambda}(0) T_{E}\right)^{P_{\lambda}+2}(F)\right\|_{n+1, E+\lambda \mu_{0}} \leqq M\|F\|_{n, E+\lambda \mu_{0}}
$$

for all $F \in H_{n, E+\lambda \mu_{0}}, \lambda$ with $|\lambda|$ sufficiently small and some $M>0$ independent of $\lambda$. Thus we conclude from (2.5) that (4.11) holds in general and the proof of Lemma 3.1 follows immediately from the definition of $\xi_{\lambda, E}$.

\section{References}

1. Anderson, P.: Phys. Rev. 109, 1492 (1958)

2. Carmona, R.: Random Schrödinger operators. Proceedings of the Ecole d'ete de probabilitie XIV Saint-Flour. 1984. Lect. Notes in Math. Vol. 1180. Berlin, Heidelberg, New York: Springer 1986 
3. Klein, A., Lacroix, J., Speis, A.: Regularity of the density states in the Anderson model on a strip for potentials with singular continuous distributions. J. Stat. Phys. 57, 65-88 (1988)

4. Kappus, M., Wegner, F.: Z. Phys. B45, 16 (1981)

5. Thouless, D.: In: Ill-Condensed Matter. Baliam R., Maynard R., Toulouse G., (eds.), Amsterdam: North-Holland 1979

6. Derrida, B., Gardner, E.: J. Phys. (Paris) 45, 1283 (1984)

7. Bovier, A., Klein, A.: Weak disorder expansions of the invariant measure for the onedimensional Anderson model. J. Stat. Phys. 51, 501-517 (1988)

8. Campanino, M., Klein, A.: Anomalies in the one dimensional Anderson model at weak disorder. Commun. Math. Phys. 130, 441-456 (1990)

9. Speis, A.: Instability of the anomalies in the one dimensional Anderson model at weak disorder. J. Stat. Phys. 63, 617-641 (1991)

10. Derrida, B., Zanon, N.: Weak disorder expansions of Lyapunov exponents in a degenerate case. J. Stat. Phys., (to appear)

11. Klein, A., Speis, A.: Smoothness at the density of states in the Anderson model on a one dimensional Anderson model. Ann. Phys. 183, 352-398 (1988)

12. Reed, M., Simon, B.: Methods of modern mathematical physics. II. Fourier analysis, selfadjointness. New York: Academic Press 1978

13. Klein, A., Lacroix, J., Speis, A.: Localization for the Anderson model on a strip with singular potentials. J. Funct. Anal. 94, 135-155 (1990)

14. Klein, A., Landau, L., Perez, F.: Suppersymmetry and the Parisi-Sourlas dimensional reduction: a rigorous proof. Commun. Math. Phys. 93, 459-482 (1984)

Communicated by T. Spencer 